\title{
Combination of glycosphingosomes and liposomal doxorubicin shows increased activity against dimethyl- $\alpha$-benzanthracene-induced fibrosarcoma in mice
}

\author{
This article was published in the following Dove Press journal: \\ International Journal of Nanomedicine \\ 7 October 2015 \\ Number of times this article has been viewed
}

\author{
Masood A Khan' \\ Ahmed N Aljarbou ${ }^{2}$ \\ Yousef H Aldebasi' \\ Mohammed S Alorainy ${ }^{3}$ \\ Arif Khan' \\ 'College of Applied Medical Sciences, \\ ${ }^{2}$ College of Pharmacy, ${ }^{3}$ College \\ of Medicine, Qassim University, \\ Buraydah, Saudi Arabia
}

\begin{abstract}
The present study aimed to assess the antitumor effect of glycosphingolipid-incorporated liposomes (glycosphingosomes) in combination with liposomal doxorubicin (Lip-Dox) in a mouse model of fibrosarcoma. Glycosphingosomes were prepared by incorporating glycosphingolipids isolated from Sphingomonas paucimobilis into the liposomes of 1,2-dipalmitoyl-sn-glycero-3-phosphocholine, cholesterol, and cardiolipin. Tumors were induced by administering dimethyl- $\alpha$-benzanthracene, and tumor-bearing mice were treated with various formulations of Dox, including free Dox, Lip-Dox, or glycosphingosomes + Lip-Dox. Mice were observed for 90 days to monitor their survival and tumor size. Free Dox, but not Lip-Dox or a combination of glycosphingosomes and Lip-Dox, caused the substantial depletion of leukocytes and significantly increased the levels of lactate dehydrogenase and creatinine kinase in mice. Tumor-bearing mice treated with a combination of glycosphingosomes and Lip-Dox showed restricted tumor growth and increased survival when compared to those treated with free Dox or Lip-Dox. The results of the present study suggest that a combination of glycosphingosomes and Lip-Dox may prove to be very effective in the treatment of tumors.
\end{abstract}

Keywords: glycosphingolipids, NKT cells, tumors, chemotherapy

\section{Introduction}

Anticancer chemotherapy has been associated with extensive toxic manifestations in treated patients, and the potential for chemotherapy to deplete the immune system and interfere with immunosurveillance has been a major concern. ${ }^{1-3}$ The emergence of drug-resistant cancers is posing serious challenges for the treatment of malignant diseases. $^{4-6}$

Various immune cells, such as natural killer (NK) cells and cytotoxic T lymphocytes, play a protective role in suppressing the development and progression of tumors. ${ }^{7,8} \mathrm{NK}$ $\mathrm{T}$ (NKT) cells, a subset of T cells, act as a bridge between innate and adaptive immune responses, because they secrete enormous amounts of cytokines upon activation with specific lipid ligands. ${ }^{9}$ We have previously shown that type I NKT cells have an antitumor immune response, and their adoptive transfer reduces the incidence of B-cell lymphoma in type I NKT cell-deficient mice. ${ }^{10}$ Invariant NKT (iNKT) cell-deficient mice showed susceptibility to methylcholanthrene-induced sarcomas and B16F10 melanoma tumors. ${ }^{11}$ Moreover, the liver-derived iNKT cells were able to reverse the methylcholanthreneinduced effects during the early stages of sarcomas. ${ }^{12}$ The activation of NKT-cell function using specific ligands holds a therapeutic role in the treatment of various diseases,
Correspondence: Masood A Khan College of Applied Medical Sciences, Qassim University, Buraydah 5I452, Saudi Arabia

Tel +966507059437

Fax+966I6 3801628

Email a_khan@qu.edu.sa 
including cancer, as well as infectious and autoimmune diseases. ${ }^{13,14} \alpha$-Galactosylceramide ( $\alpha$-GalCer), a glycolipid extracted from the marine sponge Agelas mauritiana, can stimulate NKT cells to produce enormous amounts of both Th1 and Th2 cytokines in a CD1d-dependent way. ${ }^{14}$ NKT cells have shown both direct and indirect antitumor immune responses upon activation with $\alpha$-GalCer. ${ }^{15}$ Glycosphingolipids (GSLs) isolated from lipopolysaccharide-free Gram-negative bacteria Sphingomonas paucimobilis have the ability to stimulate NKT cells in a CD1d-dependent manner. ${ }^{16}$

Doxorubicin (Dox) has been in use for the treatment of a wide variety of solid and hematologic cancers. ${ }^{17,18}$ However, the use of Dox shows considerable cardiotoxicity in cancer patients. ${ }^{17,19}$ Liposomal formulations of Dox have shown superior antitumor activity and less cardiac toxicity by altering the pharmacokinetics and tissue distribution of the drug. ${ }^{18-20}$ Considering the role of iNKT cells in antitumor immunity, we propose the combined use of a novel liposomal formulation, composed of NKT cell-stimulating GSLs, with Dox liposomes to treat tumor-bearing mice.

\section{Materials and methods}

Liposomal-grade 1,2-dipalmitoyl-sn-glycero-3-phosphocholine (DPPC), cholesterol, and 1,1',2,2'-tetramyristoyl cardiolipin were purchased from Avanti Polar Lipids (Alabaster, AL, USA). High-performance liquid chromatography-grade chloroform and methanol were purchased from Thermo Fisher Scientific (Waltham, MA, USA). Doxrubicin was purchased from Tocris Bioscience (Bristol, UK). Dimethyl- $\alpha$-benzanthracene (DMBA) was purchased from Santa Cruz Biotechnology (Dallas, TX, USA). Lactate dehydrogenase (LDH) and creatinine kinase $(\mathrm{CK})$ kits were purchased from BioVision (Milpitas, CA, USA). Nutrient broth was purchased from HiMedia Laboratories (Mumbai, India). S. paucimobilis (ATCC 10829) was obtained from the American Type Culture Collection (Manassas, VA, USA). Female BALB/C mice (8-10 weeks of age) were obtained from the animal house facility of King Saud University, Riyadh, Saudi Arabia. The techniques used to bleed, inject, and kill the mice were approved by the Animal Ethics Committee of the College of Applied Medical Sciences, Qassim University, Buraydah, Saudi Arabia.

\section{Isolation of GSLs from S. paucimobilis}

GSLs were isolated from $S$. paucimobilis as described previously. ${ }^{16}$ Bacteria were grown in the nutrient broth, and after being washed in phosphate-buffered saline (PBS), the bacterial pellet was collected and lyophilized. The lyophilized bacteria were homogenized in methanol before adding chloroform to a 2:1 ratio of chloroform and methanol.
The lipid extract was separated from the nonlipid residue by centrifugation. The nonlipid part was reextracted with chloroform:methanol (1:1 v:v) as described previously. The lipid extracts were combined, dried by flash evaporation under vacuum, and the chloroform:methanol (2:1 vol:vol) soluble fraction was used as a crude lipid extract. The crude lipid extract was reconstituted using warm methanol containing $0.5 \mathrm{~N} \mathrm{KOH}$ at $37^{\circ} \mathrm{C}$ for 4 hours to remove phospholipids.

\section{Preparation of glycosphingosomes and doxorubicin liposomes}

Liposomes were prepared with commercial lipids with or without GSLs from S. paucimobilis. DPPC, cholesterol, and 1,1',2,2'-tetramyristoyl cardiolipin (molar ratios 10:6.8:1) with or without GSLs were dissolved in a mixture of chloroform and methanol $(2: 1)$. The solvents were evaporated under nitrogen gas to form a thin lipid layer. The lipid film was placed under high vacuum before hydration. The film was hydrated in PBS to maintain a final GSL concentration of $100 \mu \mathrm{g} / \mathrm{mL}$. The hydrated suspension was sonicated in a bath-type sonicator and lyophilized to form dried reconstituted vesicles (DRVs).

To prepare Lip-Dox, DRVs with or without GSLs were resuspended with Dox $(1 \mathrm{mg} / \mathrm{mL})$ in sterile normal saline. The drug-to-lipid molar ratio was taken as 1:10. The mixture of Dox and DRVs was hydrated followed by brief sonication to obtain the liposomal formulation. The unincorporated Dox was separated from the Lip-Dox by being passed through a Sephadex G-75 column. The liposomal formulation was extruded ten times through $100 \mathrm{~nm}$ polycarbonate filters with an extrusion device (Avanti Polar Lipids). The amount of Dox encapsulated in the liposomes was determined by fluorescence measurement (470 nm excitation $580 \mathrm{~nm}$ emission). The entrapment efficiency of Dox in liposomes was found to be $80 \% \pm 5 \%$.

\section{In vitro toxicity of free doxorubicin, Lip-Dox, or a combination of glycosphingosomes and Lip-Dox on human erythrocytes}

Human blood collected in an acid citrate dextrose solution was centrifuged to separate red blood cells (RBCs). RBCs were washed and suspended in isotonic buffer $(20 \mathrm{mM}$ phosphate buffer, $150 \mathrm{mM} \mathrm{NaCl}, \mathrm{pH} 7.4$ ) to form $50 \%$ hematocrit. Free Dox, Lip-Dox, or a combination of glycosphingosomes and Lip-Dox was added to the RBCs at various doses $(0,5$, 10 , and $20 \mu \mathrm{g} / \mathrm{mL}$ ) of Dox. The final volume was made up to $1 \mathrm{~mL}$ with PBS. The mixtures were incubated at $37^{\circ} \mathrm{C}$ for 1 hour and centrifuged at 1,500 rpm. The supernatants were analyzed for the release of hemoglobin at $576 \mathrm{~nm}$. RBCs 
treated with $1 \%$ Triton $\mathrm{X}-100$ were considered the positive control (100\% lysis). Percentage hemolysis was calculated by the following equation:

$$
\text { Percentage hemolysis }=100(\mathrm{Abs}-\mathrm{Ab} 0 / \mathrm{Ab} 100-\mathrm{Ab} 0)
$$

where $\mathrm{Abs}$ and $\mathrm{Ab0}$ are absorbance of the sample and the negative control, and Ab100 is absorbance of the positive control.

\section{In vivo toxicity of free doxorubicin, Lip-Dox, or a combination of glycosphingosomes and Lip-Dox}

To determine the toxicity of free Dox, Lip-Dox, or a combination of glycosphingosomes and Lip-Dox, mice were injected with a single dose of 1,5 , and $10 \mathrm{mg} / \mathrm{kg}$ of Dox through intraperitoneal routes. The control mice received either saline or blank liposomes. On day 4 post-drug administration, blood was drawn from the mice to count the total leukocytes. The qualitative changes in the leukocytes were also analyzed by staining the blood picture with Giemsa stain. Dox-induced cardiotoxicity was assessed by determining the levels of serum LDH and CK using specific kits.

\section{Release of doxorubicin from liposomal formulation in the presence or absence of glycosphingosomes}

To analyze the effect of glycosphingosomes on the release of Dox from the liposomal formulation, the latter was incubated with the serum in the presence or absence of glycosphingosomes. The incubation mixture, which consisted of 1 volume of Dox liposomes with 9 volumes of human serum, was incubated at $37^{\circ} \mathrm{C}$ for $1,2,3$, and 4 hours. The mixture was centrifuged at 5,000 rpm for 15 minutes. The amount of the drug released from the liposomes was calculated as a percentage of total liposomal Dox added to the reaction mixture.

\section{Induction of tumors in mice}

DMBA was dissolved in sesame oil, and BALB/C mice were orally administered with a weekly dose of $500 \mu \mathrm{g} / \mathrm{mouse}$ for 4 weeks, as described previously. ${ }^{21}$ After $16-18$ weeks, more than $80 \%$ of the mice had developed palpable tumors.

\section{Treatment schedule}

Mice were regularly observed to monitor tumor growth by measuring Vernier calipers. Chemotherapy was started when the mean tumor size reached $150-200 \mathrm{~mm}^{3}$. The 1 st day of chemotherapy was considered day 0 . Doses of $3 \mathrm{mg} / \mathrm{kg}$ and $5 \mathrm{mg} / \mathrm{kg}$, chosen to treat tumor-bearing mice, were based on the results of Dox toxicity. Each group of mice received chemotherapy through the intraperitoneal route once a week continuously for 3 weeks.

Mice were divided into nine groups, and each group comprised ten mice: 1) untreated control; 2) sham liposomes; 3) glycosphingosomes; 4) free Dox $-3 \mathrm{mg} / \mathrm{kg}$; 5) sham liposomes + Lip-Dox $-3 \mathrm{mg} / \mathrm{kg}$; 6) glycosphingosomes + Lip-Dox $-3 \mathrm{mg} / \mathrm{kg}$; 7) free Dox - $5 \mathrm{mg} / \mathrm{kg}$; 8) sham Lip + Lip-Dox - $5 \mathrm{mg} / \mathrm{kg}$; and 9) glycosphingosomes + Lip-Dox $-5 \mathrm{mg} / \mathrm{kg}$.

Tumor sizes were measured every other day according to the following formula:

$$
\mathrm{V}=\mathrm{D} \times \mathrm{d}^{2} \times \pi / 6
$$

where $\mathrm{V}$ is tumor volume, $\mathrm{D}$ is biggest dimension, and $\mathrm{d}$ is smallest dimension. The efficacy of the chemotherapy was assessed by closely monitoring the survival rate and the growth of untreated or treated groups of mice.

\section{Statistical analysis}

Mouse survival was analyzed by the log-rank test using the Kaplan-Meier curve. The sizes of tumors were compared by one-way analysis of variance using Prism software version 6.0 (GraphPad Software Inc, La Jolla, CA, USA).

\section{Results Free doxorubicin caused toxicity to human erythrocytes}

Among the drug formulations used in the present study, free Dox caused the hemolysis of erythrocytes in a dose-dependent manner. The incorporation of Dox in DPPC liposomes (LipDox) resulted in the diminution of its toxicity to a very low level. Glycosphingosome formulation caused no toxicity, and did not increase the toxicity of Lip-Dox to the erythrocytes. Free Dox at a dose of $20 \mu \mathrm{g} / \mathrm{mL}$ caused about $20 \%$ hemolysis of the erythrocytes, whereas Lip-Dox or a combination of LipDox and glycosphingosomes caused $2.8 \%$ and $3 \%$ hemolysis at the same doses, respectively (Figure 1). Therefore, the use of Lip-Dox alone or in combination with glycosphingosomes did not result in significant toxicity to the erythrocytes when compared to free Dox at the same dose (Figure 1).

\section{Administration of free doxorubicin, but not Lip-Dox or a combination of Lip- Dox and glycosphingosomes, caused substantial depletion of white blood cells}

The use of chemotherapeutic anticancer drugs has been associated with leukopenia in treated patients. Mice were injected with a single dose of 1,5 , and $10 \mathrm{mg} / \mathrm{kg}$ of the Dox 


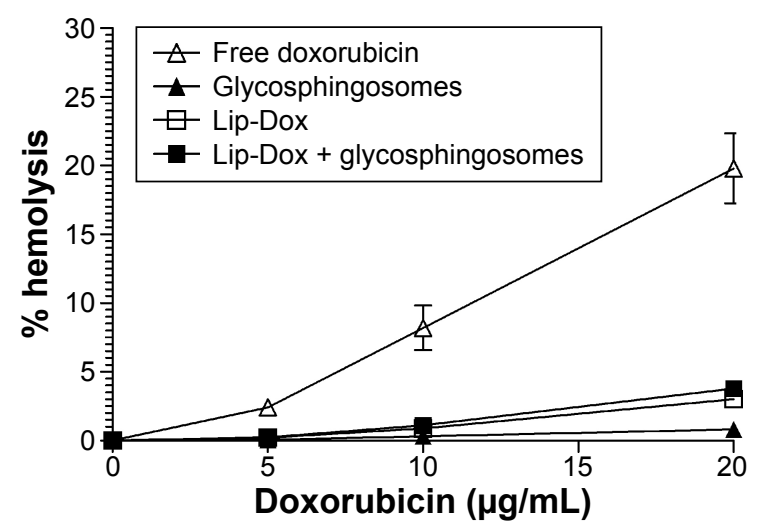

Figure I Hemolysis of erythrocytes by various formulations of doxorubicin. Notes: Human erythrocytes ( $50 \%$ hematocrit) were incubated with various doses $(0,5,10$, and $20 \mu \mathrm{g} / \mathrm{mL})$ of free doxorubicin, Lip-Dox, or Lip-Dox + glycosphingosomes for I hour at $37^{\circ} \mathrm{C}$. Data presented as means \pm standard deviation of three different experimental values.

Abbreviation: Lip-Dox, liposomal doxorubicin.

formulations, including free Dox or Lip-Dox. Free Dox caused the reduction of leukocytes in a dose-dependent manner, and the group of mice injected with free Dox at a dose of $10 \mathrm{mg} / \mathrm{kg}$ showed $\sim 84 \%$ reduction in leukocytes (Figure 2A), whereas the groups of mice injected with the same dose of Lip-Dox or a combination of Lip-Dox and glycosphingosomes showed $\sim 43 \%$ and $\sim 38 \%$ reductions in the leukocyte count, respectively (Figure 2A).

In addition to the quantitative changes, the leukocytes from the mice injected with free Dox $(10 \mathrm{mg} / \mathrm{kg})$ also showed qualitative changes, such as abnormal morphology and disorganized nuclei, as well as dispersed chromatin strands and granules, whereas leukocytes from the mice injected with Lip-Dox or a combination of Lip-Dox and glycosphingosomes at the same dose showed fewer toxic manifestation (Figure 2B).

\section{Mice injected with free doxorubicin, but not Lip-Dox or a combination of Lip-Dox and glycosphingosomes, showed increased cardiac toxicity}

The toxicity of Dox formulations, including free Dox or Lip-Dox, was assessed by determining the serum levels of LDH and CK. Mice treated with free Dox at a dose of $10 \mathrm{mg} / \mathrm{kg}$ showed a $108 \%$ increase in the level of LDH and a $188 \%$ increase in the level of CK when compared to the normal control (Figure 3A and B), whereas the groups of mice treated with Lip-Dox showed $38 \%$ and $52 \%$ increases, respectively, and treatment with a combination of Lip-Dox and glycosphingosomes resulted in $22 \%$ and $32 \%$ increases, respectively (Figure $3 \mathrm{~A}$ and B).

\section{The presence of glycosphingosomes did not alter the stability of doxorubicin liposomes}

To assess the effect of glycosphingosomes on the stability of Dox liposomes, Dox liposomes were incubated with serum in the absence or presence of glycosphingosomes. Glycosphingosomes did not significantly alter the release of Dox from the liposomal formulation. The release of Dox was $\sim 23 \%$ from Lip-Dox, whereas the release of Dox from the same
A

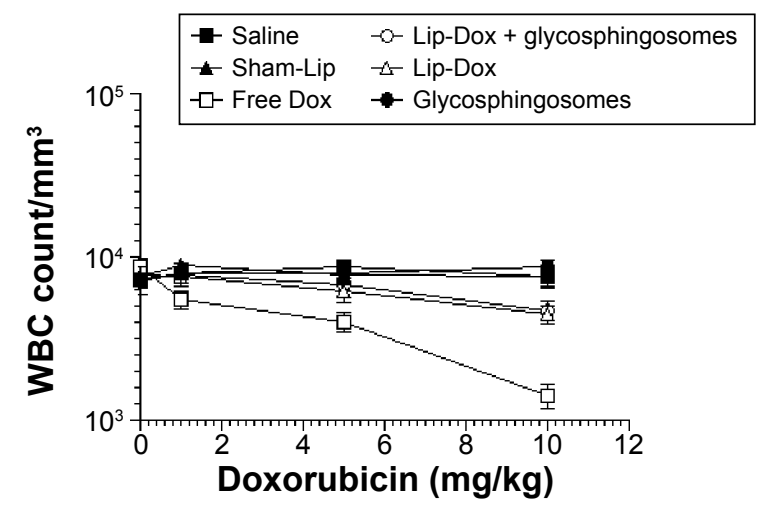

B

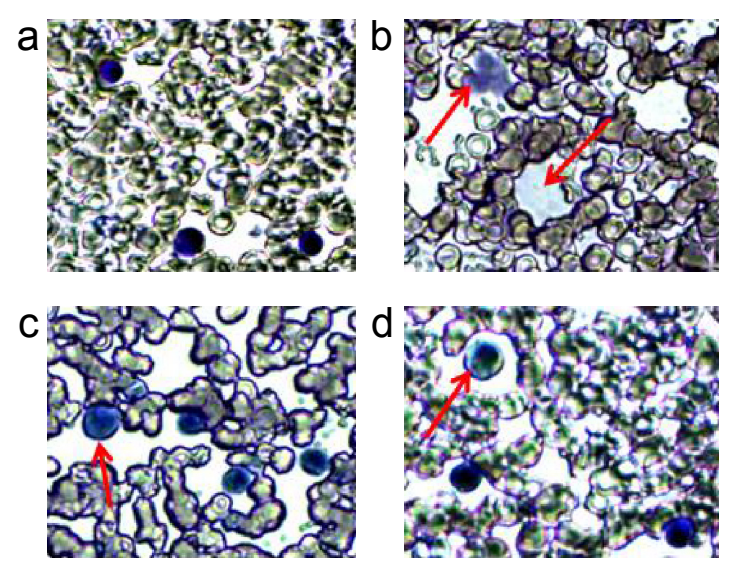

Figure 2 Doxorubicin-induced toxicity to leukocytes in mice.

Notes: The BALB/C mice were injected with various doses $(0,1,5$, and $10 \mathrm{mg} / \mathrm{kg}$ ) of doxorubicin formulations via the intraperitoneal route. (A) On day 4 post-drug administration, blood was taken and the numbers of leukocytes counted. Data presented as means \pm standard deviation of values from three different mice. (B) Using $40 \times$ magnification, qualitative changes in leukocytes from the groups of mice injected with doxorubicin formulations at a dose of $10 \mathrm{mg} / \mathrm{kg}$ were observed. (a) Leukocytes from normal control mice; (b) free doxorubicin-injected mice showed disintegrated leukocytes with dispersed chromatin strands and granules (as shown by arrow); (c) mice treated with Lip-Dox showed normal and apoptotic leukocytes (arrow indicates the leukocyte undergoing apoptosis with pyknosis and cytoplasmic vacuolations); (d) mice injected with a combination of Lip-Dox and glycosphingosomes also showed normal leukocytes and leukocytes undergoing apoptosis (the arrow indicates a leukocyte showing marked cytoplasmic vacuolation).

Abbreviations: Lip-Dox, liposomal doxorubicin; WBC, white blood cell. 
A

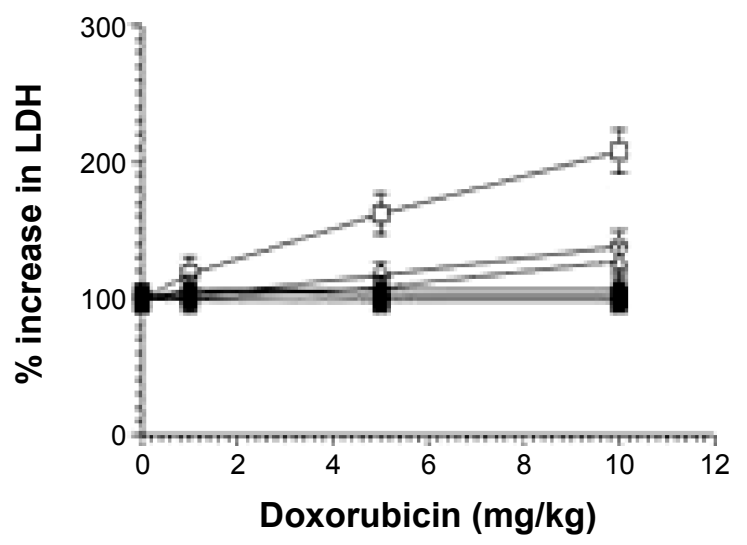

B

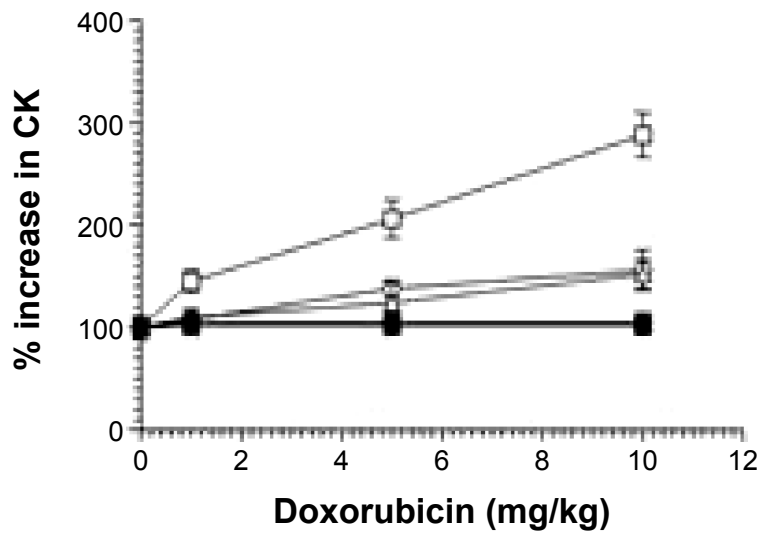

Saline $\square-$ Free Dox $\rightarrow$ Sham-Lip $\rightarrow$ Lip-Dox $\rightarrow$ GSL-Lip $-0-$ GSL-Lip-Dox

Figure 3 Coadministration of glycosphingosomes did not contribute to doxorubicin-induced cardiac toxicity in mice.

Notes: BALB/C mice were injected with various doses $(0,1,5$, and $10 \mathrm{mg} / \mathrm{kg}$ ) of free doxorubicin, Lip-Dox, or a combination of Lip-Dox and glycosphingosomes. On day 4 , blood was drawn and levels of (A) LDH or (B) CK measured. Data presented as means \pm standard deviation of three different experimental values.

Abbreviations: Lip-Dox, liposomal doxorubicin; GSL, glycosphingolipid; LDH, lactate dehydrogenase; CK, creatine kinase.

liposomal formulation in the presence of glycosphingosomes was found to be $\sim 19 \%$ (Figure 4 ).

\section{Coadministration of Lip-Dox and glycosphingosomes effectively restricted tumor growth}

To determine the antitumor effect of glycosphingosomes, LipDox with or without glycosphingosomes was administered to fibrosarcoma-bearing mice at doses of $3 \mathrm{mg} / \mathrm{kg}$ or $5 \mathrm{mg} / \mathrm{kg}$ of the drug. Therapy was started when tumors attained a size of about $150-200 \mathrm{~mm}^{3}$. Fibrosarcoma-bearing mice were treated weekly with a single dose of free Dox or Lip-Dox for 3 weeks. Among all formulations, the use of a combination of

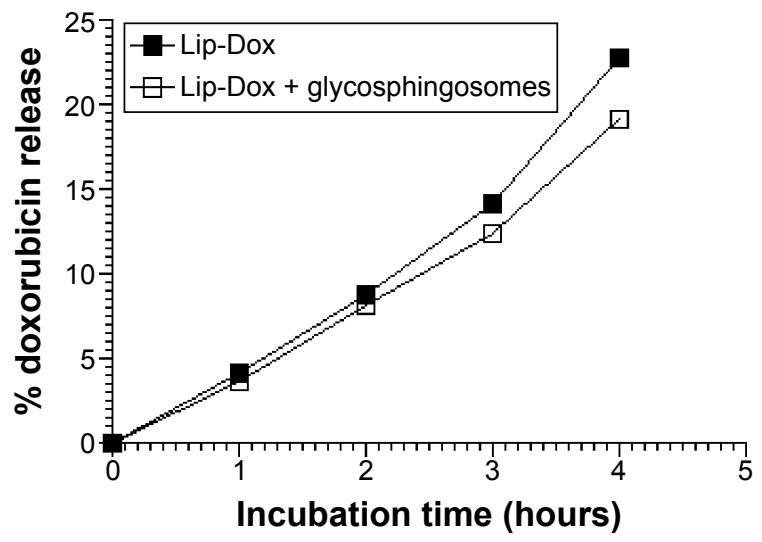

Figure 4 The presence of glycosphingosomes did not alter the release of doxorubicin from liposomes.

Notes: The release of doxorubicin from liposomes was determined in the absence or presence of glycosphingosomes by incubation of Lip-Dox in human serum, as described in the Materials and methods section. Data presented as means \pm standard deviation of three different experimental values.

Abbreviation: Lip-Dox, liposomal doxorubicin.
Lip-Dox and glycosphingosomes most effectively restricted tumor growth when compared to free Dox or a combination of Lip-Dox and sham liposomes (no glycosphingosomes) at the same doses (Figure 5). Interestingly, the group of mice treated with glycosphingosomes alone (no chemotherapy) also showed delayed tumor growth when compared to the sham liposome-treated group (Figure 5).

\section{Fibrosarcoma-bearing mice treated with a combination of Lip-Dox and glycosphingosomes showed greater survival}

Besides assessing the effect of chemotherapy on the size of the tumors, the effect of various formulations of Dox was also observed on the survival of tumor-bearing mice. Consistent with its effect on tumor growth, treatment with a combination of Lip-Dox and glycosphingosomes imparted the highest survival to fibrosarcoma-bearing mice (Figure 6). Fibrosarcoma-bearing mice treated with a combination of Lip-Dox and glycosphingosomes at doses of $3 \mathrm{mg} / \mathrm{kg}$ and $5 \mathrm{mg} / \mathrm{kg}$ of Dox showed $30 \%$ and $50 \%$ survival, respectively, on day 90 after the start of the chemotherapy (Figure 6), whereas all mice treated with free Dox (at doses of $3 \mathrm{mg} / \mathrm{kg}$ and $5 \mathrm{mg} / \mathrm{kg})$ died within 90 days $(P=0.0013, P=0.0055$, respectively). All mice treated with Lip-Dox ( $3 \mathrm{mg} / \mathrm{kg}$ ) died within 90 days, whereas the group of mice treated with LipDox at a dose of $5 \mathrm{mg} / \mathrm{kg}$ showed $20 \%$ survival (Figure 6). The group of mice treated with glycosphingosomes (no chemotherapy) showed prolonged survival (mean survival 51 days), whereas mice treated with sham liposomes showed a mean 


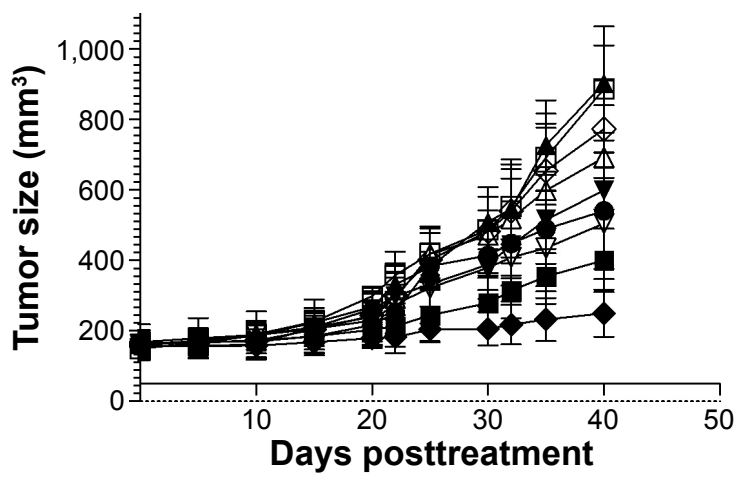

Figure 5 A combination of glycosphingosomes and liposomal doxorubicin (LipDox) effectively restricted dimethyl- $\alpha$-benzanthracene-induced tumor growth. Notes: Treatment of tumor-bearing mice was initiated when tumor size reached a volume of approximately $150-200 \mathrm{~mm}^{3}$. Tumor-bearing mice treated with a combination of glycosphingosomes and Lip-Dox $(5 \mathrm{mg} / \mathrm{kg})$ showed restricted or delayed growth of tumors when compared to treatment with Lip-Dox or free doxorubicin. Untreated control $(\square)$, sham liposomes $(\boldsymbol{\Delta})$, glycosphingosomes $(\boldsymbol{\nabla})$, free doxorubicin - $3 \mathrm{mg} / \mathrm{kg}(\diamond)$, sham liposomes + Lip-Dox - $3 \mathrm{mg} / \mathrm{kg}(\bullet)$, glycosphingosomes + Lip-Dox $-3 \mathrm{mg} / \mathrm{kg}(\boldsymbol{\square})$, free doxorubicin $-5 \mathrm{mg} / \mathrm{kg}(\triangle)$, sham liposomes + Lip-Dox $-5 \mathrm{mg} / \mathrm{kg}(\nabla)$, and glycosphingosomes + Lip-Dox $-5 \mathrm{mg} / \mathrm{kg}(\diamond)$. Glycosphingosomes + Lip-Dox versus sham Liposomes + Lip-Dox, $P=0.0192$; glycosphingosomes + Lip-Dox versus free doxorubicin, $P=0.0160$. Data presented as means \pm standard deviation ( $n=10$ mice at the initiation of therapy; the number varied at later time points due to some mortalities).

survival of only 32 days $(P=0.0266)$. The mean survival $(\sim 51$ days) for the group of mice treated with glycosphingosomes was also greater than the mean survival (41 days) for the group treated with free Dox at a dose of $3 \mathrm{mg} / \mathrm{kg}(P=0.0238)$.

\section{Discussion}

The cytotoxic effects of anticancer agents in suppressing the immune system and interfering with immunosurveillance are major challenges in anticancer therapy. The side effects of

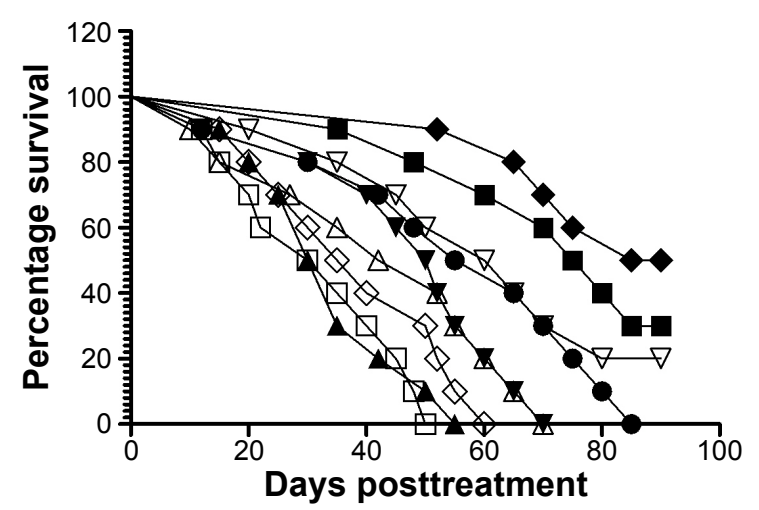

Figure 6 Tumor-bearing mice treated with a combination of glycosphingosomes and liposomal doxorubicin (Lip-Dox) showed increased survival.

Notes: Dimethyl- $\alpha$-benzanthracene-induced fibrosarcoma-bearing mice were treated with various formulations of doxorubicin, including free doxorubicin, LipDox or a combination of glycosphingosomes and Lip-Dox for 3 weeks. The Ist day of treatment was considered day 0 . The mortality and morbidity of tumor-bearing mice were observed for 90 days postchemotherapy. Untreated control ( $\square$ ), sham liposomes $(\boldsymbol{\Delta})$, glycosphingosomes $(\boldsymbol{\nabla})$, free doxorubicin $-3 \mathrm{mg} / \mathrm{kg}(\diamond)$, sham liposomes + Lip-Dox - $3 \mathrm{mg} / \mathrm{kg}(\mathbf{O})$, glycosphingosomes + Lip-Dox - $3 \mathrm{mg} / \mathrm{kg}(\boldsymbol{\square})$, free doxorubicin - $5 \mathrm{mg} / \mathrm{kg}(\triangle)$, sham liposomes + Lip-Dox - $5 \mathrm{mg} / \mathrm{kg}(\nabla)$, glycosphingosomes + Lip-Dox $-5 \mathrm{mg} / \mathrm{kg}(\bullet)$. anticancer drugs can potentially be reduced by loading them in delivery systems. ${ }^{20}$ Liposomes not only help to reduce the toxicity of encapsulated drugs but also have a better chance of combating drug-resistant tumors. ${ }^{22,23}$

NKT cells, a subset of $\mathrm{T}$ cells, act as a bridge between the innate and adaptive immune responses. ${ }^{24}$ Upon stimulation with specific lipid ligands, NKT cells secrete enormous amounts of cytokines and play protective roles against various tumors. ${ }^{24,25}$ Abnormalities in the number and functions of type I NKT cells have been found in patients with malignant diseases. ${ }^{26}$ The GSLs from S. paucimobilis activate type I NKT cells, and we have previously shown that type I NKT cells play a protective role against a B-cell lymphoma. ${ }^{10}$ GSL-incorporated liposomes possess strong immunoadjuvant potential. ${ }^{27}$ In the present study, we showed that a combination of glycosphingosomes and Lip-Dox was found to be highly effective in the treatment of tumor-bearing mice.

The use of Dox has been associated with severe toxic manifestations, whereas the liposomal formulations of the drug substantially reduce the drug's toxicity. ${ }^{18,28,29}$ The administration of Dox has been shown to cause toxicity and the depletion of leukocytes. ${ }^{31,32}$ The results of the present study showed that free Dox induces both quantitative and qualitative changes in the leukocytes. The group of mice treated with free Dox exhibited a substantial reduction in leukocyte count compared to the groups injected with Lip-Dox or a combination of Lip-Dox and glycosphingosomes. Dox induces apoptosis in the leukocytes by interfering with the DNA-replication machinery, or by inhibiting the synthesis of DNA and RNA. ${ }^{28}$ This is also shown by the results of the present study, which showed extensive damage and a wide range of toxicity in the leukocytes of mice treated with free Dox. Dox can be well tolerated in liposomal form, as the mice from the groups treated with Lip-Dox showed significantly less toxicity. Interestingly, the addition of glycosphingosomes to Lip-Dox did not contribute to any toxic manifestations in the treated mice. Free Dox-induced severe cardiac toxicity, as shown by the high levels of LDH and CK in the sera of the treated mice. Conversely, the groups of mice treated with LipDox in the presence or absence of glycosphingosomes showed reduced levels of LDH and CK in their sera. The preferential uptake of liposomal drugs by hepatic and splenic macrophages may lead to the reduced availability of the drug to the heart and other organs, which ultimately helps to reduce drug-induced toxicity. ${ }^{33}$ Surprisingly, the addition of glycosphingosomes further reduced Dox-induced toxicity. This may be attributed to the counter effects of the GSL-induced production of Th1 cytokines to Dox-induced Th2 cytokines, as Th2 cytokines contribute to Dox-induced experimental glomerulosclerosis. ${ }^{34}$ 
The stability of Lip-Dox in the absence or presence of glycosphingosomes was determined. There was $\sim 23 \%$ release of Dox from Lip-Dox after 4 hours of incubation with $90 \%$ (v:v) serum at $37^{\circ} \mathrm{C}$, whereas in the presence of glycosphingosomes there was $\sim 19 \%$ release of Dox from the same liposomal formulation. The reduced release of Dox from the liposomes in the presence of glycosphingosomes may be attributed to redistribution of some of the Dox from the liposomes to the glycosphingosomes or binding of a fraction of the released Dox to the glycosphingosomes.

Lip-Dox has been found to be effective in the treatment of various cancers, especially since liposomes increase the therapeutic index of the drug. The addition of immunostimulatory molecules to liposomes may further increase the activity of the loaded drug. Our earlier study showed that the incorporation of tuftsin increased the antitumor activity of etoposide. ${ }^{21}$ NKT cells play a role in immunosurveillance against various tumors and cancers. ${ }^{35,36}$ Type I NKT cells can directly induce cytotoxicity against tumor cells by releasing perforin/granzyme B lytic granules, or may achieve this aim indirectly by activating other immune cells, such as NK cells and dendritic cells. ${ }^{37}$ Earlier studies have also shown the protective role of the NKT-cell agonist $\alpha$-GalCer in combination with chemotherapy against osteosarcoma and multiple myeloma. ${ }^{38,39}$

GSLs from S. paucimobilis are CD1d-specific ligands for type I NKT cells. ${ }^{16}$ The combination of glycosphingosomes and Lip-Dox was the most effective in restricting tumor growth or increasing the survival of tumor-bearing mice over the other formulations of Dox, including the combination of sham liposomes and Lip-Dox or free Dox. An increased antitumor effect of a combination of glycosphingosomes and Lip-Dox can be attributed to the presence of glycosphingosomes in the preparation. GSLs stimulate type I NKT cells, which results in the secretion of enormous amounts of both Th1 and Th 2 cytokines. ${ }^{27}$ Furthermore, IFN $\gamma$, a Th1 cytokine, can induce the tumor-killing function of dendritic cells, primarily through the production of nitric oxide. ${ }^{40}$

The use of soluble $\alpha$-GalCer is generally associated with NKT-cell anergy. NKT cells agonists presented by CD1dexpressing B-cells to NKT cells have been shown to induce NKT-cell anergy in mice. ${ }^{41}$ In this context, $\alpha$-GalCer induced the activation of anergic NKT cells, and it did not protect against B16-tumor metastases. ${ }^{41}$ Some of tumors have been shown to evade NKT-cell functions by shedding the inhibitory glycolipids. ${ }^{42}$ Interestingly, the advantage of using nanoparticle formulations of $\alpha$-GalCer is that they can be administered repeatedly to activate the function of NKT cells if required, without inducing anergy. ${ }^{43}$ A novel liposomal formulation of GSLs can consistently stimulate type I NKT cells without inducing anergy, and it may prove very effective when used in combination with chemotherapy. To elucidate the effect, we prepared glycosphingosomes from the GSLs of S. paucimobilis. Unlike soluble GSLs, which are presented by B cells, liposomal GSLs are preferentially taken up and presented by dendritic cells and macrophages. ${ }^{43}$ Glycosphingosomes can consistently stimulate NKT cells without inducing anergy.

In the present study, we achieved the highest level of activity using a combination of glycosphingosomes and Lip-Dox against DMBA-induced fibrosarcoma. During the treatment period, there were no marked differences in tumor growth among treatment groups. Unlike the combination of glycosphingosomes and Lip-Dox, which restricted tumor growth even after the treatment period, free Dox restricted it only during the first 3 weeks. Moreover, the fibrosarcoma-bearing mice treated with glycosphingosomes (no chemotherapy) showed a greater survival rate than those treated with sham liposomes or free Dox, which indicates the important role of type I NKT cells against tumors. The activation of NKT cells stimulates the components of innate and adaptive immunity by virtue of their secreting both Th1 ad Th2 cytokines. Keeping in mind the role of NKT cells in antitumor immunity, the use of liposomal GSLs can be very effective when used in combination with chemotherapy to treat tumor-bearing patients. This approach can also be utilized to treat drug-resistant tumors.

\section{Acknowledgment}

This research was supported by a National Science, Technology and Innovation Plan (NSTIP) grant (10-NAN1225-09), Qassim University, Buraydah, Saudi Arabia.

\section{Disclosure}

The authors report no conflicts of interest in this work.

\section{References}

1. Tian S, Hirshfield KM, Jabbour SK, et al. Serum biomarkers for the detection of cardiac toxicity after chemotherapy and radiation therapy in breast cancer patients. Front Oncol. 2014;4:277.

2. Mackall CL. T-cell immunodeficiency following cytotoxic antineoplastic therapy: a review. Stem Cells. 2000;18(1):10-18.

3. Mackall CL, Fleisher TA, Brown MR, et al. Lymphocyte depletion during treatment with intensive chemotherapy for cancer. Blood. 1994;84(7): 2221-2228.

4. Kim JJ, Tannock IF. Repopulation of cancer cells during therapy: an important cause of treatment failure. Nat Rev Cancer. 2005;5(7): 516-525.

5. Ramos P, Bentires-Alj M. Mechanism-based cancer therapy: resistance to therapy, therapy to resistance. Oncogene. Epub 2014 Sep 29.

6. Ellis LM, Hicklin DJ. Resistance to targeted therapies: refining anticancer therapy in the era of molecular oncology. Clin Cancer Res. 2009;15(24): 7471-7478

7. Swann JB, Smyth MJ. Immune surveillance of tumors. J Clin Invest. 2007;117(5):1137-1146. 
8. Smyth MJ, Thia KY, Street SE, et al. Differential tumor surveillance by natural killer (NK) and NKT cells. J Exp Med. 2000;191(4):661-668.

9. Brutkiewicz RR, Sriram V. Natural killer T (NKT) cells and their role in antitumor immunity. Crit Rev Oncol Hematol. 2002;41(3):287-298.

10. Renukaradhya GJ, Khan MA, Vieira M, Du W, Gervay-Hague J, Brutkiewicz RR. Type I NKT cells protect (and type II NKT cells suppress) the host's innate antitumor immune response to a B-cell lymphoma. Blood. 2008;111(12):5637-5645.

11. Crowe NY, Smyth MJ, Godfrey DI. A critical role for natural killer $\mathrm{T}$ cells in immunosurveillance of methylcholanthrene-induced sarcomas. J Exp Med. 2002;96(1):119-127.

12. Smyth MJ. NK cells and NKT cells collaborate in host protection from methylcholanthrene-induced fibrosarcoma. Int Immunol. 2008; 20(4):631.

13. James EE, Andrew JK, Webb TJ. Raising the roof: the preferential pharmacological stimulation of Th1 and Th2 responses mediated by NKT cells. Med Res Rev. 2014;34(1):45-76.

14. Brutkiewicz RR. CD1d ligands: the good, the bad, and the ugly. J Immunol. 2006;177(2):769-775.

15. Morita M, Motoki K, Akimoto K, et al. Structure-activity relationship of $\alpha$-galactosylceramides against B16-bearing mice. J Med Chem. 1995;38(12):2176-2187.

16. Sriram V, Du W, Gervay-Hague J, Brutkiewicz RR. Cell wall glycosphingolipids of Sphingomonas paucimobilis are CD1d-specific ligands for NKT cells. Eur J Immunol. 2005;35(6):1692-1701.

17. Carvalho C, Santos RX, Cardoso S, et al. Doxorubicin: the good, the bad and the ugly effect. Curr Med Chem. 2009;16(25):3267-3285.

18. Rivankar S. An overview of doxorubicin formulations in cancer therapy. J Cancer Res Ther. 2014;10(4):853-858.

19. Singal PK, Iliskovic N. Doxorubicin-induced cardiomyopathy. NEngl J Med. 1998;339(13):900-905.

20. Tahover E, Patil YP, Gabizon AA. Emerging delivery systems to reduce doxorubicin cardiotoxicity and improve the therapeutic index: focus on liposomes. Anticancer Drugs. 2015;26(3):241-258.

21. Khan A, Khan AA, Dwivedi V, Ahmad MG, Hakeem S, Owais M. Tuftsin augments antitumor efficacy of liposomized etoposide against fibrosarcoma in Swiss albino mice. Mol Med. 2007;13(5-6):266-276.

22. Markan JL, Rekechenetskiy A, Holler E, Ljubimova JY. Nanomedicine therapeutic approaches to overcome cancer drug resistance. Adv Drug Deliv Rev. 2013;65(13-14):1866-1879.

23. Candoni A, Michelutti A, Simeone E, Damiani D, Baccarani M, Fanin R. Efficacy of liposomal daunorubicin and cytarabine as reinduction chemotherapy in relapsed acute lymphoblastic leukemia despite expression of multidrug resistance-related proteins. Eur J Haematol. 2006;77(4): 293-299.

24. Brutkiewicz RR, Lin Y, Cho S, Hwang YK, Sriram V, Roberts TJ. CD1d-mediated antigen presentation to natural killer T (NKT) cells. Crit Rev Immunol. 2003;23(5-6):403-419.

25. Robertson FC, Berzofsky JA, Terabe M. NKT cell networks in the regulation of tumor immunity. Front Immunol. 2014;5:543.

26. Nowak M, Schmidt-Wolf IG. Natural killer T cell subsets in cancer, functional defects in prostate cancer and implications for immunotherapy. Cancers (Basel). 2011;3(3):3661-3675.
27. Inoue J, Ideue R, Takahashi D, Kubota M, Kumazawa Y. Liposomal glycosphingolipids activate natural killer T cell-mediated immune responses through the endosomal pathway. J Control Release. 2009;133(1): $18-23$.

28. Richardson DS, Johnson SA. Anthracyclines in haematology: preclinical studies, toxicity and delivery systems. Blood Rev. 1997;11(4): 201-223.

29. Allen TM, Martin FJ. Advantages of liposomal delivery systems for anthracyclines. Semin Oncol. 2004;31(6):5-15.

30. Lorusso V, Manzione L, Silvestris N. Role of liposomal anthracyclines in breast cancer. Ann Oncol. 2007;18 Suppl 6:vi70-vi73.

31. Fu X, Kong L, Tang M, et al. Protective effect of ocotillol against doxorubicin-induced acute and chronic cardiac injury. Mol Med Rep. 2014;9(1):360-364.

32. Datta S, Parajuli N, Tymoszuk P, et al. Replenishment of the B cell compartment after doxorubicin-induced hematopoietic toxicity is facilitated by STAT1. J Leukoc Biol. 2014;95(6):853-866.

33. Khan MA, Owais M. Toxicity, stability and pharmacokinetics of amphotericin B in immunomodulator tuftsin-bearing liposomes in a murine model. J Antimicrob Chemother. 2006;58(1):125-132.

34. Pereira RL, Reis VO, Semedo P, et al. Invariant natural killer T cell agonist modulates experimental focal and segmental glomerulosclerosis. PLoS One. 2012;7(3):e32454.

35. Vivier E, Ugolini S, Blaise D, Chabannon C, Brossay L. Targeting natural killer cells and natural killer T cells in cancer. Nat Rev Immunol. 2012;12(4):239-252.

36. Swann JB, Coquet JM, Smyth MJ, Godfrey DI. CD1-restricted T cells and tumor immunity. Curr Top Microbiol Immunol. 2007;314:293-323.

37. Hayakawa Y, Takeda K, Yagita H, et al. IFN- $\gamma$-mediated inhibition of tumor angiogenesis by natural killer T-cell ligand, $\alpha$-galactosylceramide. Blood. 2002;100(5):1728-1733.

38. Fallarini S, Paoletti T, Orsi Battaglini N, Lombardi G. Invariant NKT cells increase drug-induced osteosarcoma cell death. Br J Pharmacol. 2012;167(7):1533-1549.

39. Richter J, Neparidze N, Zhang L, et al. Clinical regressions and broad immune activation following combination therapy targeting human NKT cells in myeloma. Blood. 2013;121(3):423-430.

40. LaCasse CJ, Janikashvili N, Larmonier CB, et al. Th-1 lymphocytes induce dendritic cell tumor killing activity by an IFN- $\gamma$-dependent mechanism. J Immunol. 2011;187(12):6310-6317.

41. Parekh VV, Wilson MT, Olivares-Villagómez D, et al. Gycolipid antigen induces long term natural killer T cell anergy in mice. J Clin Invest. 2005;115(9):2572-2583.

42. Sriram V, Cho S, Li P, et al. Inhibition of glycolipid shedding rescues recognition of a CD1+ T cell lymphoma by natural killer T (NKT) cells. Proc Natl Acad Sci U S A. 2002;99(12):8197-8202.

43. Thapa P, Zhang G, Xia C, et al. Nanoparticle formulated alphagalactosylceramide activates NKT cells without inducing anergy. Vaccine. 2009;27(25-26):3484-3488.
International Journal of Nanomedicine

\section{Publish your work in this journal}

The International Journal of Nanomedicine is an international, peerreviewed journal focusing on the application of nanotechnology in diagnostics, therapeutics, and drug delivery systems throughout the biomedical field. This journal is indexed on PubMed Central, MedLine, CAS, SciSearch $\AA$, Current Contents ${ }^{\circledR} /$ Clinical Medicine,

\section{Dovepress}

Journal Citation Reports/Science Edition, EMBase, Scopus and the Elsevier Bibliographic databases. The manuscript management system is completely online and includes a very quick and fair peer-review system, which is all easy to use. Visit http://www.dovepress.com/ testimonials.php to read real quotes from published authors. 